\title{
3017- Basic and clinical immunology - 3017: A functional polymorphism in IL-5 receptor alpha may influence asthma severity in patients with aspirin-exacerbated respiratory disease
}

\author{
Purevsuren Losol", Seung-Hyun Kim, Yoo Seob Shin, Young Min Ye, Hae-Sim Park
}

From 2nd WAO International Scientific Conference (WISC 2012)

Hyderabad, India. 6-9 December 2012

\section{Background}

Eosinophilic infiltration into the tissues of the airway is a central feature of aspirin-exacerbated respiratory disease (AERD). Eosinophil activation and survival are profoundly influenced by IL- 5 and its receptor, IL-5R. In patients susceptible to allergic disorders, IL $5 R A$ polymorphisms have been reported, however, an association with AERD remains unclear. We hypothesise that the presence of IL5RA polymorphisms will increase the genetic susceptibility to AERD.

\section{Methods}

We recruited 139 AERD patients, 171 aspirin-tolerant asthma (ATA) patients and 160 normal controls. IL5RA polymorphisms $(-5993 \mathrm{G}>\mathrm{A},-5567 \mathrm{C}>\mathrm{G},-5091 \mathrm{G}>\mathrm{A})$ were genotyped and functional studies were assessed by luciferase reporter assay and electrophoretic mobility shift assay (EMSA). Asthma severity was classed into three groups according the FEV1 predicted value at the enrolment period following the GINA guidelines.

\section{Results}

The genotype frequency of $-5993 \mathrm{G}>\mathrm{A}$ was significantly associated with asthma severity in AERD patients $(P<0.05)$. The frequency of the minor allele at the IL5RA $-5993 \mathrm{G}>$ A polymorphism was significantly higher in moderate and severe patients when compared with mild patients (severe $v s$. mild, $P=0.032$ for the dominant model; severe $v s$. moderate, $P=0.041$ for the co-dominant model, and $P=0.012$ for the dominant model). Moderate and severe patients in the AERD group, carrying the AA genotype at $-5993 \mathrm{G}>\mathrm{A}$, had a significantly higher prevalence of specific IgE to staphylococcal superantigens than those with the GG/GA genotype $(P=0.005)$. In vitro, the $-5993 \mathrm{~A}$ allele had a higher promoter activity compared to the -5993G allele in human mast cells (HMC-1) $(P=0.030)$ and eosinophilic cells (HL-60) $(P=0.013)$. In EMSA, a $-5993 \mathrm{~A}$ probe produced a specific shifted band than the $-5993 \mathrm{G}$ had. The shifted band produced by the $-5993 \mathrm{~A}$ probe was not visible in the presence of the nonlabeled $-5993 \mathrm{G}$ probe but remained visible in the presence of the nonlabeled -5993A probe.

\section{Conclusions}

A functional polymorphism in IL5RA could contribute to eosinophil and mast cell activation in AERD, and aggravate asthma severity along with specific IgE responses to staphylococcal superantigens.

Published: 23 April 2013

doi:10.1186/1939-4551-6-S1-P193

Cite this article as: Losol et al.: 3017- Basic and clinical immunology 3017: A functional polymorphism in IL-5 receptor alpha may influence asthma severity in patients with aspirin-exacerbated respiratory disease. World Allergy Organization Journal 2013 6(Suppl 1):P193. 